\title{
miR-3187-3p enhances migration and invasion by targeting PER2 in head and neck squamous cell carcinomas
}

\author{
Lei Xiao ${ }^{1,2,3 *}$, Chao Liu1,2,3*, Shuiting Zhang1,2,3, Yuanzheng Qiu 1,2,3,4, Donghai Huang1,2,3, Diekuo Zhang1,2,3, \\ Huihong Chen ${ }^{1,2,3}$, Hang Ling $1,2,3$, Yong Liu1, $1,3,4 \bowtie$, Xin Zhang1,2,3, $₫$ \\ 1. Department of Otolaryngology Head and Neck Surgery, Xiangya Hospital, Central South University, 87 Xiangya Road, Changsha, Hunan 410008 , China \\ 2. Otolaryngology Major Disease Research Key Laboratory of Hunan Province, 87 Xiangya Road, Changsha, Hunan 410008, China. \\ 3. Clinical Research Center for Pharyngolaryngeal Diseases and Voice Disorders in Hunan Province, 87 Xiangya Road, Changsha, Hunan 410008, China. \\ 4. National Clinical Research Center for Geriatric Disorders (Xiangya Hospital), 87 Xiangya Road, Changsha, Hunan 410008, China. \\ * These two authors contributed equally to this paper.
}

$\triangle$ Corresponding authors: Yong Liu and Xin Zhang. Address: Department of Otolaryngology Head and Neck Surgery, Xiangya Hospital, Central South University, 87 Xiangya Road, Changsha, Hunan 410008, China. Tel.: +86073184327469; Fax: +86073184327469. E-mail: liuyongent@csu.edu.cn (Y.L); xinzhang@csu.edu.cn (X. Z).

(c) The author(s). This is an open access article distributed under the terms of the Creative Commons Attribution License (https://creativecommons.org/licenses/by/4.0/). See http://ivyspring.com/terms for full terms and conditions.

Received: 2021.01.24; Accepted: 2021.06.07; Published: 2021.06.26

\begin{abstract}
Invasion and metastasis are major contributors to treatment failure in patients with head and neck squamous cell carcinomas (HNSCCs) and microRNAs (miRNAs) are reported to play important roles in tumor progression. Our study therefore try to find the crucial miRNAs and reveal their molecular and functional mechanisms involved in migration and invasion of HNSCCs. Through The Cancer Genome Atlas (TCGA) data analysis, we screened out miR-3187-3p and its biological function and specific mechanism were further analyzed. The wound-healing and transwell invasion assay demonstrated that miR-3187-3p promoted the capacity of migration and invasion of HNSCCs in vitro. Luciferase reporter assays showed that PER2 was a direct target of miR-3187-3p, which could reverse the effect of miR-3187-3p in HNSCCs. Furthermore, we found that miR-3187-3p / PER2 axis activated the Wnt / $\beta$-catenin signaling pathway in HNSCCs. Altogether, our study indicated that miR-3187-3p enhanced migration and invasion by targeting PER2 in HNSCCs.
\end{abstract}

Key words: Head and neck squamous cell carcinoma; miR-3187-3p; invasion; metastasis

\section{Introduction}

Head and neck squamous cell carcinomas (HNSCCs), derived from the epithelial cells of the upper aerodigestive tract (the oral cavity, the nasopharynx, the oropharynx, the hypopharynx and the larynx) [1], have approximately 800,000 new cases yearly with more than 50\% 5-year mortality [2]. Despite recent advances in treatment strategy, especially targeted therapy [3] and checkpoint therapy [4], the prognosis for patients with HNSCCs has remained uniformly poor, which is definitely due to invasion and metastasis of tumor for a large part [5]. It is therefore important and necessary to reveal the molecular mechanisms underlying invasion and metastasis of HNSCCs, which can offer vital countermeasures and predicted biomarkers for the improvement of prognosis of patients with HNSCCs.

First discovered in 1993 [6], microRNAs (miRNAs) are a family of $\sim 22$ nucleotide (nt) long small non-coding RNAs that function by silencing their target gene [7]. It is clear now that miRNAs are involved in the regulation of most protein-coding transcripts activities, contributing to the normal cellular and physiological function of human beings [8]. Meanwhile, the aberrant expression of miRNAs inevitably could regulate diverse malignant biological behavior, including proliferation, metastasis, radioresistance, immunosuppression and so on [9-14]. A mass of researches have revealed that miRNAs dysregulation can contribute to tumor progression in numerous kinds of cancers, including HNSCCs [15, 
16]. Hence adjustment of specific dysregulation miRNAs represents a promising treatment avenue toward HNSCCs [8].

Previously, through analyzing The Cancer Genome Atlas (TCGA) database, we had screened a number of differentially expressed miRNAs associated with metastasis of HNSCCs, in which miR-3187-3p was included [16]. miR-3187-3p was reported to exert tumor-promoting role in colorectal cancer and non-small cell lung cancer [17, 18]. However, the exact role of miR-3187-3p in HNSCC remains to be elucidated. In this study, we demonstrated that miR-3187-3p was not only an independent prognostic factor for HNSCCs patients but also tightly associated with migration and invasion traits of HNSCCs by targeting PER2 and activating the Wnt / $\beta$-catenin signaling pathway. These findings provide valuable clues to understand the molecular mechanisms involving migration and invasion of HNSCCs.

\section{Materials and methods}

\section{Cell culture}

In the study, four HNSCCs cell lines JHU011, Tu686, Tca8113, Fadu and one precancerous lesions of the oral mucosa cell line DOK were used. JHU011 cell line were kindly gifted by Dr Joseph Califanio (University of California, San Diego, USA). Tu686 cell line was kindly offered by Dr Zhuo G. Chen (Emory University Winship Cancer Institute, USA) [16]. Tca8113 and DOK cell lines were acquired from The Cell Bank of Type Culture Collection of Chinese Academy of Sciences. Fadu cell line was bought from ATCC. These cells were maintained in appropriate medium (DMEM/F12 medium, DMEM basic medium, or RPMI 1640 medium) supplemented with $10 \%$ fetal bovine serum (FBS, Biological Industries) in $5 \% \mathrm{CO}_{2}$ at $37^{\circ} \mathrm{C}$.

\section{Patient samples}

All HNSCCs tissue samples and patients' information were obtained in the same manner as our previous study [16], in which 83 cases of HNSCCs were included. The study was conducted in accordance with the Declaration of Helsinki and approved by the Research Ethics Committee of Xiangya hospital, Central South University, and written informed consents were obtained from all these patients.

\section{RNA isolation and gene expression analysis}

Total RNA was extracted using Trizol reagent (Invitrogen). First strand cDNA was synthesized with the All-in-One ${ }^{\mathrm{TM}}$ cDNA synthesis kit (GeneCopoeia). Quantitative real-time PCR (qRT-PCR) was conducted with QuantStudio 7 Flex Real-Time PCR System (ThermoFisher Scientific). Data derived from qRT-PCR were caculated using $2^{-\Delta \Delta} \mathrm{Ct}$ method and normalized to U6 for miRNA or GAPDH for mRNA. The experiments were carried out for three times to get the final average result. The primers used are listed in Supplementary Table S1.

\section{Western blotting}

Total protein was obtained using RIPA lysis buffer. Protein concentration was quantified by BCA assay. Then homogenates containing $20 \mu \mathrm{g}$ protein in SDS-PAGE sample loading buffer were loaded on an 8-12\% SDS-PAGE gels and protein were transferred to PVDF membranes after electrophoresis. Next, the membrane was blocked with NcmBlot blocking buffer (NCM Biotech, Suzhou, China) for 10min and subsequently incubated with relevant primary antibodies overnight at $4{ }^{\circ} \mathrm{C}$, followed by incubation with corresponding secondary antibody for $50 \mathrm{~min}$ at room temperature. Finally, target protein was detected using ECL substrates.

\section{Transfection}

Fadu and Tca8113 cell lines were transfected with miR-3187-3p mimics / negative control (NC) (Genepharma, Suzhou, China) following the manufacturer's protocol. JHU011 and Tu686 cell lines were transfected with miR-3187-3p inhibitors / NC (RiboBio, Guangzhou, China) according to the manufacturer's guidelines. PER2 overexpression plasmid transfection was performed using FuGENE HD Reagent (Promega).

\section{Wound-healing and transwell invasion assay}

The wound was scratched using a $200 \mu \mathrm{L}$ sterile micropipette tip when the HNSCCs cells reached near $100 \%$ confluence. The cells were continued to be cultured for $24-96 \mathrm{~h}$ without serum. The scratch wound was photographed under phase-contrast microscope, and the scratch area was assessed by ImageJ software.

As to transwell invasion assay, $150 \mu \mathrm{L}$ cell suspension $\left(1.5-2 \times 10^{4}\right)$ of serum-free medium were seeded in the top Transwell chamber, while $500 \mu \mathrm{L}$ complete medium containing $10 \%$ FBS was added in the lower chamber. After $48 \mathrm{~h}$ incubation, non-invaded cells were erased and the invaded cells were fixed and stained, and the number of invaded cells were then counted in five randomly selected area under high magnification microscope $(200 \times)$.

\section{Luciferase reporter assays}

Luciferase assays were conducted $48 \mathrm{~h}$ after co-transfection with miR-3187-3p mimic or NC in combination with wild or mutated type PER2 
plasmids (GeneCopoeia) in Fadu cells. Relative luciferase activity was accessed by normalizing the Firefly luciferase with the Renilla luciferase values using the Dual-Luciferase Reporter Assay System (Promega).

\section{Statistical analysis}

Statistical analysis was performed with SPSS21.0. Descriptive statistics were presented as the means \pm SD. Data were analyzed using appropriate statistical methods including independent $t$ tests, Wilcoxon tests and ANOVA. Kaplan-Meier method was used to plot survival curves and compared by log-rank test. $\mathrm{P}<0.05$ was considered as statistically significant.

\section{Results}

\section{Clinicopathologic features of miR-3187-3p in HNSCCs}

In our previous study, we had identified aberrant expression profile of miRNAs associated with HNSCCs metastasis, in which miR-3187-3p was included [16]. To further investigate the clinical significance of miR-3187-3p in patients with HNSCCs, the relative expression of miR-3187-3p was assessed in TCGA and GEO database using $\mathrm{R}$ package limma. miR-3187-3p was highly expressed in 525 HNSCCs samples from TCGA and 57 HNSCCs samples form GEO (GSE133632) compared with the adjacent normal tissues (Fig. 1A, B; P < 0.001). Paired cancer and corresponding paracancerous tissue in both TCGA and GEO also showed a significantly higher expression of miR-3187-3p in tumor (Fig. 1C, D; P < 0.001). Furthermore, miR-3187-3p was strongly associated with both clinical and pathologic N Status (Table 1; P < 0.001) and survival analysis revealed that HNSCCs patients with higher miR-3187-3p expression displayed a poorer prognosis than those with lower expression of miR-3187-3p (Fig. 1E, F; P < 0.05).

Besides, we collected 83 tissue samples to further analyze the clinical significance of miR-3187-3p in HNSCCs and the relative expression of miR-3187-3p was quantified by qRT-PCR. As shown in Table 2, the high expression of miR-3187-3p was closely related to the higher $\mathrm{T}$ stage, advanced clinical stage and lymph node metastasis in HNSCCs patients (Table 2; $\mathrm{P}$ $<0.05)$. Meanwhile, the prognosis of patients with high miR-3187-3p expression was significantly worse than those with low miR-3187-3p expression (Figure $1 G, P<0.001$ ), which was consistent with the results of TCGA and GEO database analysis. Collectively, these data suggest that miR-3187-3p may be a valuable prognosis biomarker for patients with HNSCCs.

Table 1. Correlations between miR-3187-3p expression and clinicopathological features in TCGA HNSCCs patients.

\begin{tabular}{llll}
\hline Clinical characteristics & Total (N) & ANOVA P-value & ANOVA FDR \\
\hline Clinical M Status & 497 & $6.21 \mathrm{e}-01$ & $9.80 \mathrm{e}-01$ \\
Clinical N Status & 500 & $\mathbf{6 . 1 7 e}-\mathbf{0 5}$ & $\mathbf{4 . 7 3 e - 0 3}$ \\
Clinical Stage & 508 & $9.93 \mathrm{e}-01$ & $9.96 \mathrm{e}-01$ \\
Clinical T Status & 506 & $9.79 \mathrm{e}-01$ & $9.91 \mathrm{e}-01$ \\
Histologic Grade & 499 & $3.85 \mathrm{e}-01$ & $6.04 \mathrm{e}-01$ \\
Pathologic M Status & 189 & $8.70 \mathrm{e}-01$ & $9.58 \mathrm{e}-01$ \\
Pathologic N Status & 422 & $\mathbf{6 . 8 0 e}-\mathbf{0 7}$ & $5.71 e-05$ \\
Pathologic Stage & 450 & $5.54 \mathrm{e}-01$ & $9.48 \mathrm{e}-01$ \\
Pathologic T Status & 460 & $3.05 \mathrm{e}-01$ & $6.41 \mathrm{e}-01$ \\
Sex & 522 & $5.92 \mathrm{e}-01$ & $8.48 \mathrm{e}-01$ \\
\hline Note: This table was obtained from the OncomiR (http://www.oncomir.org/). ${ }^{*} \mathrm{P}<$
\end{tabular}

0.05 was considered to be statistically significant (in bold and italics).

Table 2. Correlations between miR-3187-3p expression and clinicopathological parameters in HNSCCs clinical samples.

\begin{tabular}{|c|c|c|c|c|}
\hline Parameters & No. of patients & miR-3187-3p expression & $t$ value & $P$ value \\
\hline \multicolumn{5}{|l|}{ Age } \\
\hline$<59$ & 41 & $0.79 \pm 0.60$ & 0.522 & 0.603 \\
\hline$\geq 59$ & 42 & $0.73 \pm 0.34$ & & \\
\hline \multicolumn{5}{|l|}{ Gender } \\
\hline Female & 4 & $0.62 \pm 0.28$ & 0.580 & 0.564 \\
\hline Male & 79 & $0.77 \pm 0.51$ & & \\
\hline \multicolumn{5}{|l|}{ Smoking } \\
\hline Yes & 47 & $0.76 \pm 0.39$ & -0.033 & 0.974 \\
\hline No & 36 & $0.76 \pm 0.62$ & & \\
\hline \multicolumn{5}{|c|}{ Histological grade } \\
\hline $\mathrm{G} 1+\mathrm{G} 2$ & 29 & $0.78 \pm 0.63$ & 0.305 & 0.761 \\
\hline G3 & 54 & $0.75 \pm 0.41$ & & \\
\hline \multicolumn{5}{|c|}{$\mathrm{T}$ classification } \\
\hline $\mathrm{T} 1$ & 16 & $0.52 \pm 0.20$ & -2.179 & 0.032 \\
\hline $\mathrm{T} 2+\mathrm{T} 3+\mathrm{T} 4$ & 67 & $0.82 \pm 0.53$ & & \\
\hline \multicolumn{5}{|c|}{ Clinical stage } \\
\hline $\mathrm{I}+\mathrm{II}$ & 36 & $0.54 \pm 0.21$ & -3.869 & $<0.001$ \\
\hline $\mathrm{III}+\mathrm{IV}$ & 47 & $0.93 \pm 0.58$ & & \\
\hline \multicolumn{5}{|c|}{ Lymph node metastasis } \\
\hline N0 & 54 & $0.60 \pm 0.30$ & -4.394 & $<0.001$ \\
\hline $\mathrm{N}+$ & 29 & $1.06 \pm 0.64$ & & \\
\hline
\end{tabular}

\section{Overexpression of miR-3187-3p promotes HNSCCs migration and invasion}

To directly evaluate the tumor-promoting role of miR-3187-3p in HNSCCs, we quantified its expression in oral mucosa cell line DOK and HNSCCs cell lines, and found that the expression of miR-3187-3p was higher in HNSCCs cells than DOK cells (Supplementary Fig. S1). The relatively higher expression of miR-3187-3p cell lines (JHU011, Tu686) and relatively lower expression cell lines (Fadu, Tca8113) were selected for further loss and gain of function experiments, respectively. A significantly upregulated expression of miR-3187-3p was confirmed using qRT-PCR after transfection with miR-3187-3p mimics in Fadu and Tca8113 cell lines (Fig. 2A; $\mathrm{P}<0.001$ ). Wound-healing and transwell invasion assays were conducted to assess migration 
and invasion abilities of different HNSCCs cells. As shown in Fig. 2B, C, overexpression of miR-3187-3p significantly increased the migration and invasion capacities of cancer cells. Our results thus demonstrate that miR-3187-3p facilitates migration and invasion of HNSCC cells.

\section{Downexpression of $\mathrm{miR}-3187-3 p$ suppresses HNSCCs migration and invasion}

We conducted loss of function experiments to further confirm the role of miR-3187-3p in Tu686 and

A

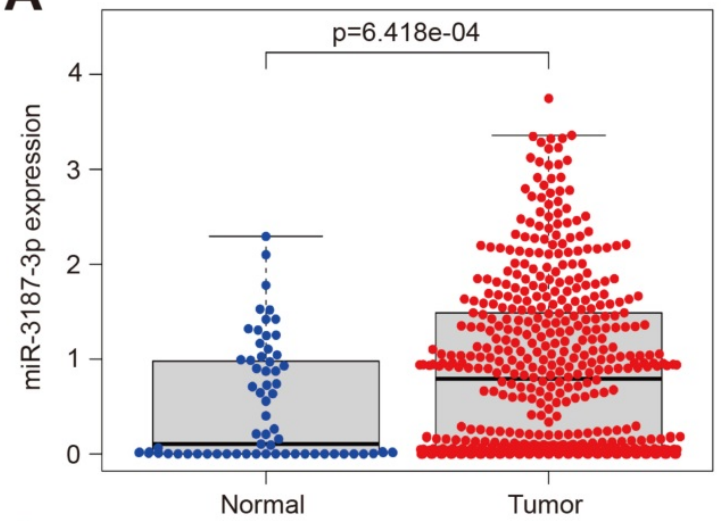

C

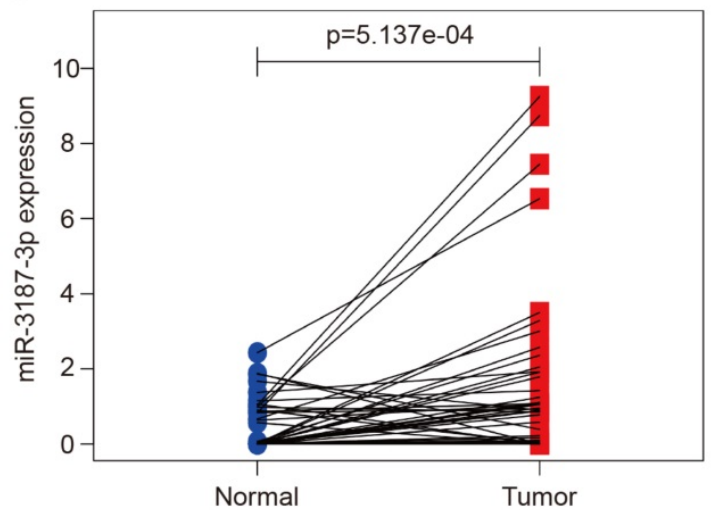

JHU011 HNSCCs cells. The expression of miR-3187-3p was successfully downregulated with inhibitors (Fig. 3A). As expected, the wound healing rates and successfully invaded cell numbers were reduced accordingly compared to the control (Fig. 3B, C), suggesting the negative regulation of tumor migratory and invasive ability by miR-3187-3p inhibition in HNSCCs.

B

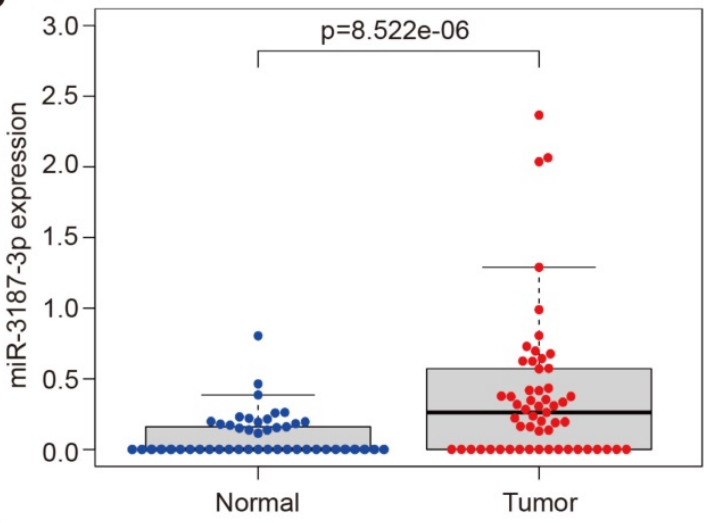

D

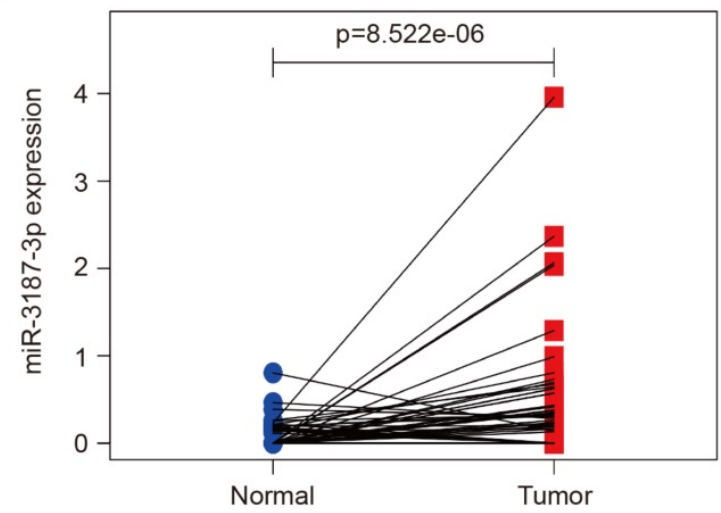

$\mathbf{E}$

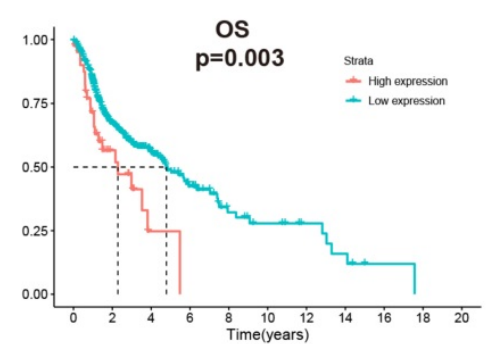

Number at risk
$\mathbf{F}$

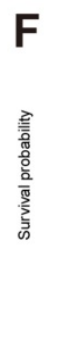

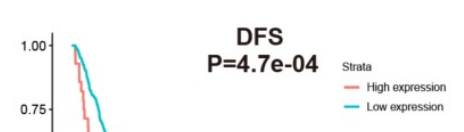

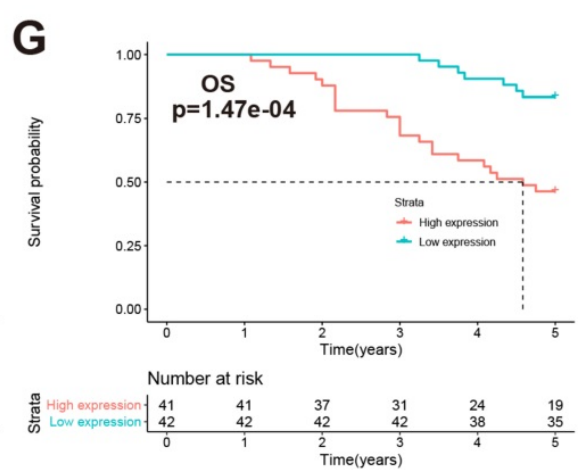

Figure 1. Clinicopathologic features of miR-31 87-3p in HNSCCs. A. The expression of miR-3187-3p in 525 HNSCCs samples and 44 normal sample in TCGA database. B. The expression of miR-3187-3p in 57 laryngeal squamous cell carcinoma (LSCC) samples and 57 adjacent normal sample in GEO database (GSE133632). C. The expression of miR-3187-3p in 43 paired TCGA tumor and corresponding adjacent noncancerous tissues. D. The expression of miR-3187-3p in LSCC tissues (57 samples) and paired adjacent normal mucosa tissues in GEO database (GSE133632). E, F. Overall survival (OS) (E) and Disease-free survival (DFS) (F) analysis was implemented in patients with HNSCCs according to the expression level of miR-3187-3p using Kaplan-Meier survival curves in TCGA data (The best cutoff was obtained through Xtile to distinguish high and low miR-3187-3p levels; cutoff $=3.9$ ). G. Kaplan-Meier analysis in patients with HNSCC between the high expression group $(n=41)$ and low expression group $(n=42)$ of miR-3187-3p. 


\section{miR-3 187-3p activates Wnt / $\beta$-catenin signaling pathway in HNSCCs}

The Wnt / $\beta$-catenin pathway was a predominant oncogenic pathway, whose activation was the characteristic of multiple malignant biobehaviors, including tumor invasion and metastasis [19-23]. In the study, we analyzed the relationship between levels of miR-3187-3p and biomarkers of Wnt / $\beta$-catenin pathway in HNSCCs cells by Western bolt. As shown in Fig. 4A, overexpressed miR-3187-3p significantly increased the protein expression of $\beta$-catenin, $p$-GSK-3 $\beta$ and c-Myc in Fadu and Tca8113 cell lines. Conversely, obvious downregulation of $\beta$-catenin, p-GSK-3 $\beta$ and c-Myc protein levels were observed by miR-3187-3p inhibition in JHU011 and Tu686 cell lines (Fig. 4B). These results indicated that miR-3187-3p activated the Wnt / $\beta$-catenin signaling pathway in HNSCCs.
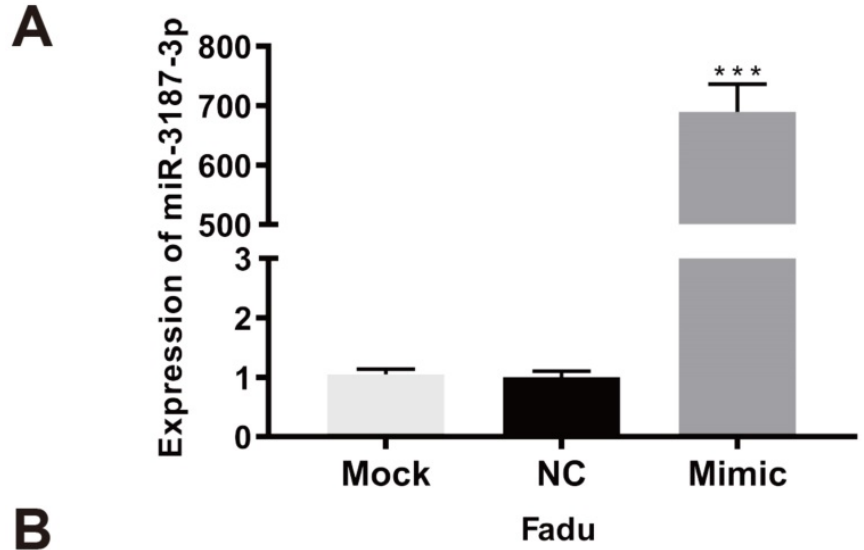

Fadu
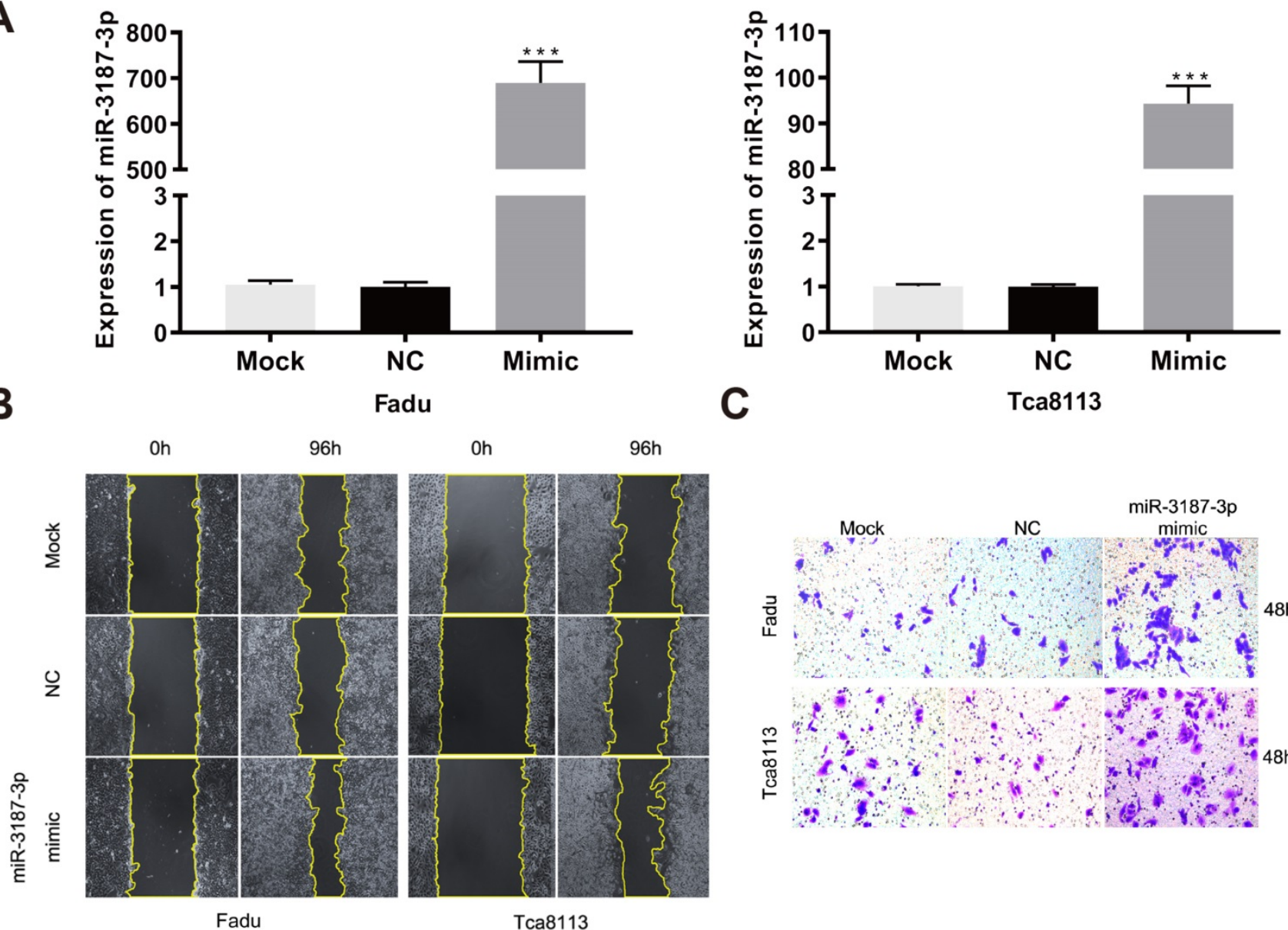

Oh

$96 \mathrm{~h}$
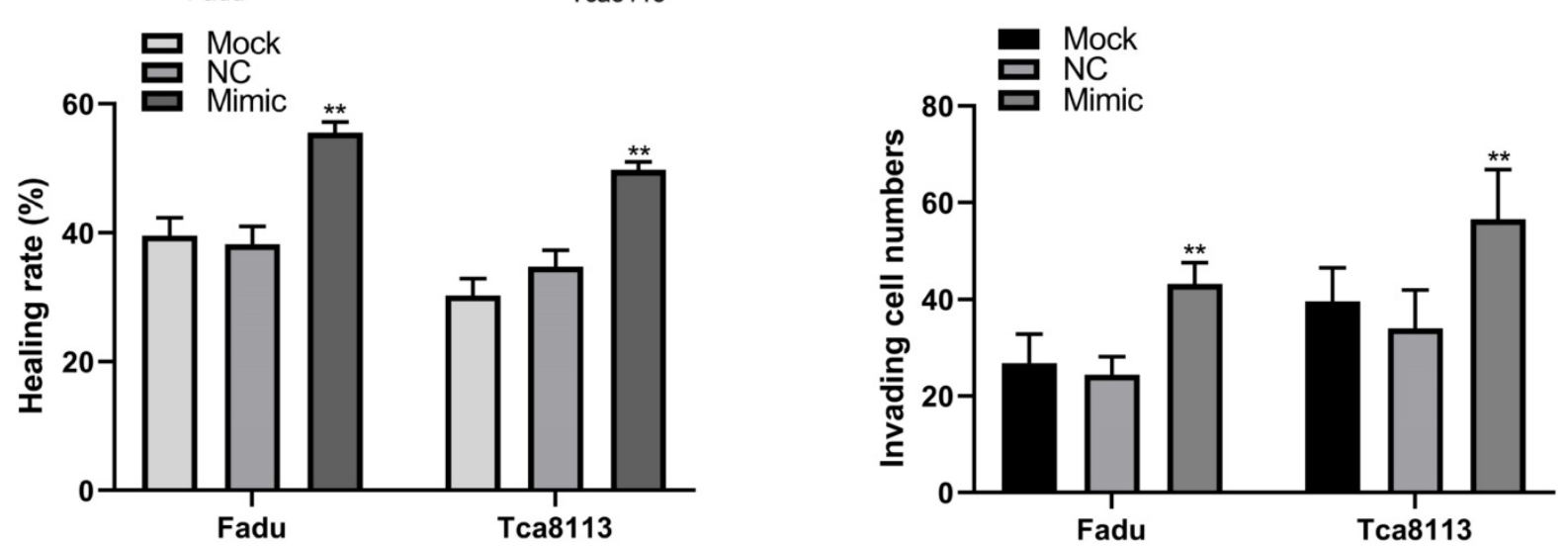

Figure 2. Overexpression of miR-3187-3p promotes HNSCCs migration and invasion. A. The expression of miR-3187-3p was measured using qRT-PCR after transfected with miR-3187-3p-mimic. B. The migration ability was assessed by the wound healing test, and the quantitative results of healing rate was determined. C. Representative images of transwell invasion assay for cells with different miR-3187-3p expression levels, and the invaded cells numbers were count. Data are presented as the mean \pm SD. Student's unpaired t-test, $* *, P<0.01 ; * * *, P<0.001$. 


\section{PER2 is a direct target of $\mathrm{miR}-3187-3 p$ in HNSCCs}

In order to determine the potential target of miR-3187-3p, five online target gene prediction algorithms (TargetScan, micro-T-CDS, miRDB, miRTarBase and miRWalk) were used. As a result, 12 possible candidate target genes were gained, including PER2 (Fig. 5A). Then we upregulated and downregulated the expression of miR-3187-3p respectively in HNSCCs cell lines and identified PER2 as the most relevant gene (Fig. 5B). In addition, PER2 exhibited an opposite expression and prognosis style in contrast to miR-3187-3p according to the HNSCCs TCGA data analysis (Fig. 5C, D). Moreover, the luciferase reporter assay successfully validated that miR-3187-3p directly bound to the 3'UTR of PER2 as expected (Fig. 5E, F). These data demonstrated that PER2 is a direct target of miR-3187-3p in HNSCCs.

A

B

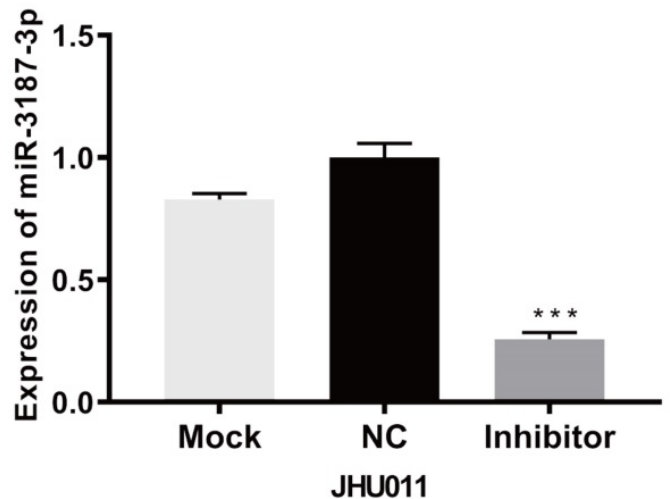

Oh

$24 \mathrm{~h}$

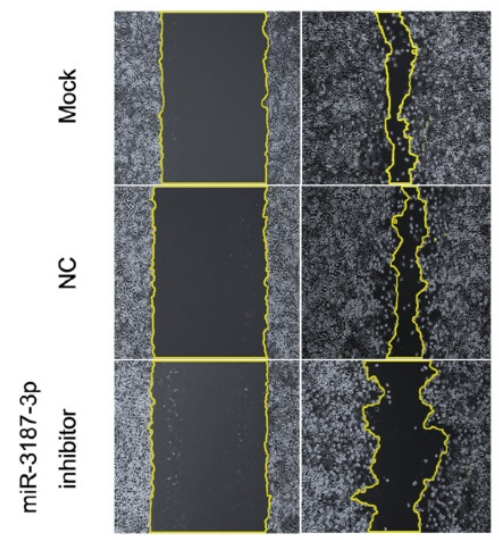

JHU011
Oh

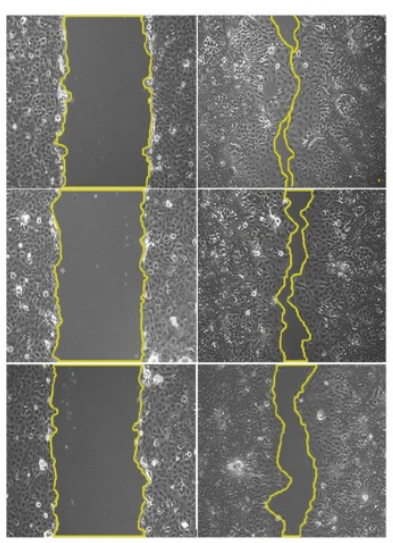

Tu686
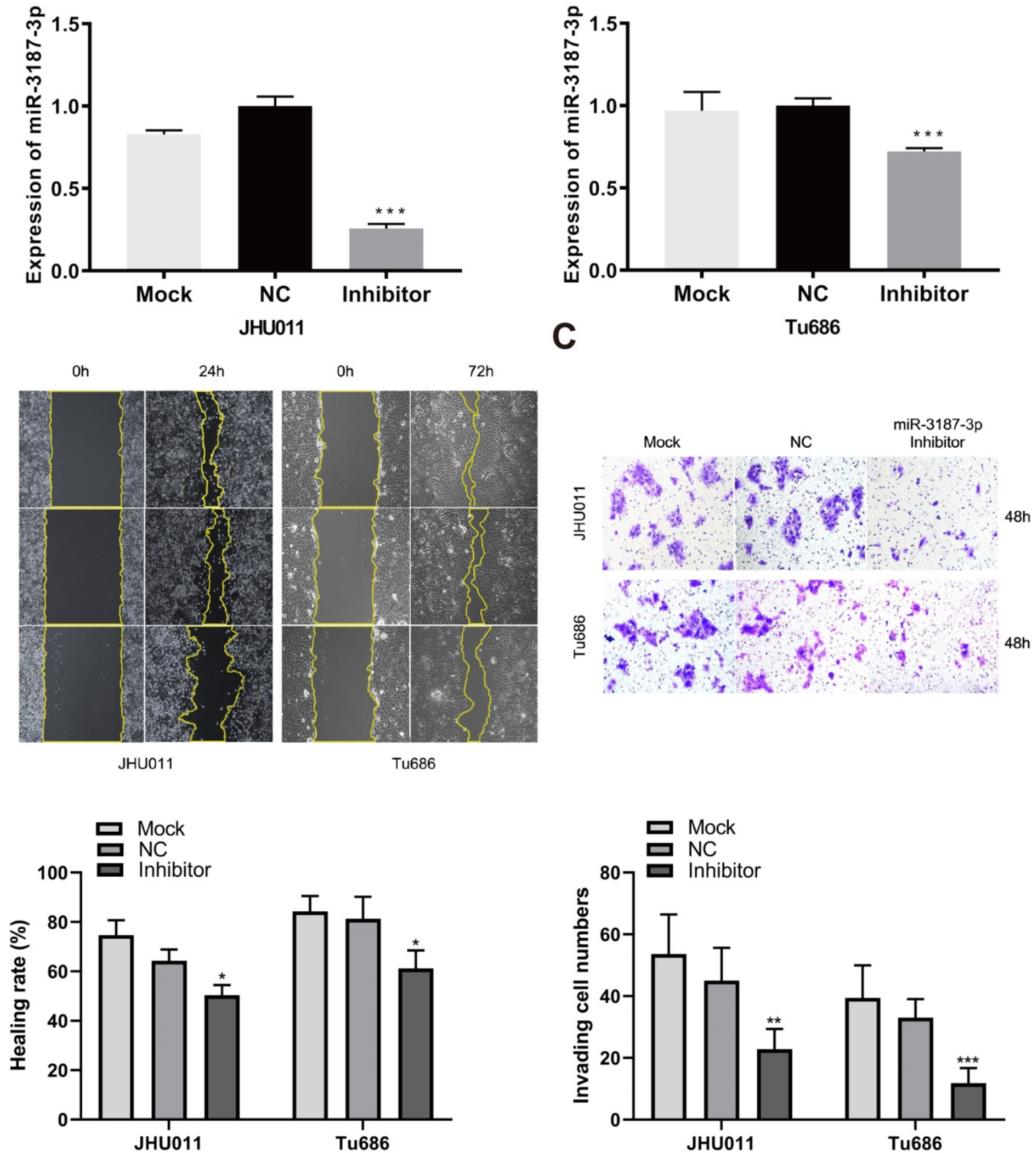

Figure 3. Downexpression of miR-3187-3p suppresses HNSCCs migration and invasion. A. The expression of miR-3187-3p in JHU011 and Tu686 cell lines was determined after transfection with miR-3187-3p inhibitor using qRT-PCR analyses. B. Wound healing tests in JHU011 and Tu686 cells indicated that cell migration ability was attenuated by the miR-3187-3p-inhibitor. C. Representative fields with invaded cells were obtained from transwell assays, and quantification of the invaded cells in different groups were compared. All data are represented as the mean \pm SD. Student's unpaired t-test, *, $\mathrm{P}<0.05 ; * *, \mathrm{P}<0.01 ; * * *, \mathrm{P}<0.001$. 


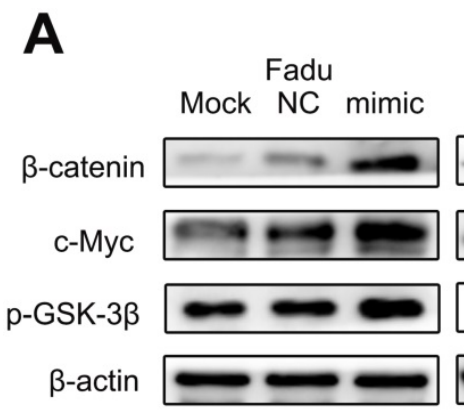

Tca8113

Mock NC mimic
B

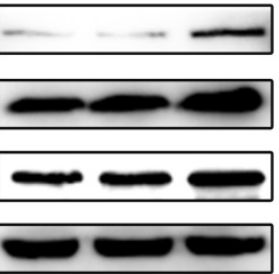

JHU011

Mock NC Inhibitor Mock NC Inhibitor
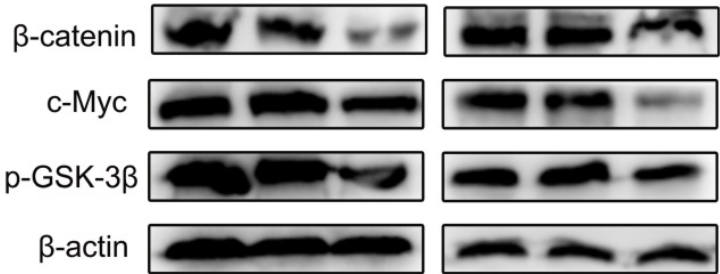

Figure 4. miR-3 187-3p activates Wnt / $\beta$-catenin signaling pathway in HNSCCs. A. The protein levels of $\beta$-catenin、p-GSK-3 $\beta$ and c-Myc were analyzed by Western blot in Fadu and Tca8113 cells transfected with miR-3187-3p mimic. B. The protein levels of $\beta$-catenin, p-GSK-3 $\beta$ and c-Myc were analyzed by Western blotting in JHU011 and Tu686 cells transfected with miR-3187-3p inhibitor. $\beta$-actin was used as a loading control.

A

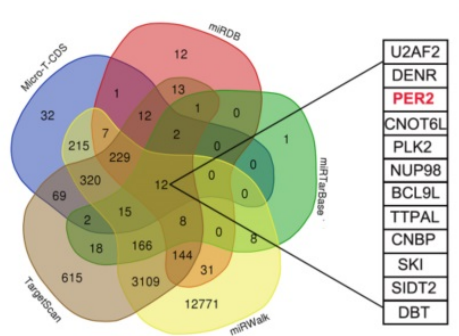

B
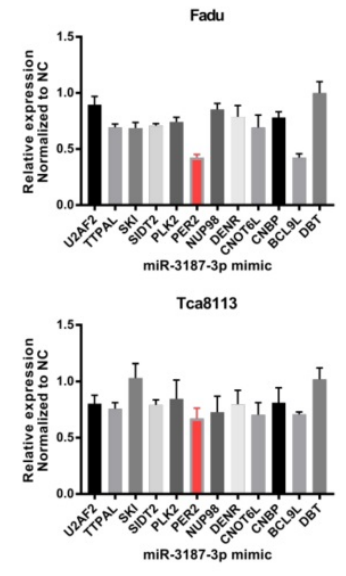

JHU011

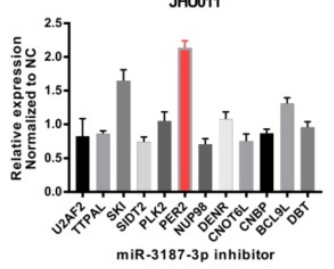

C

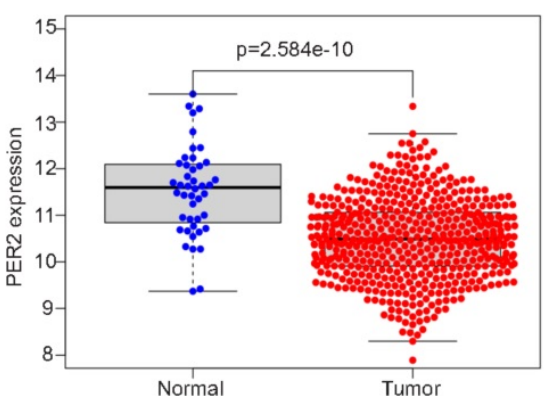

D

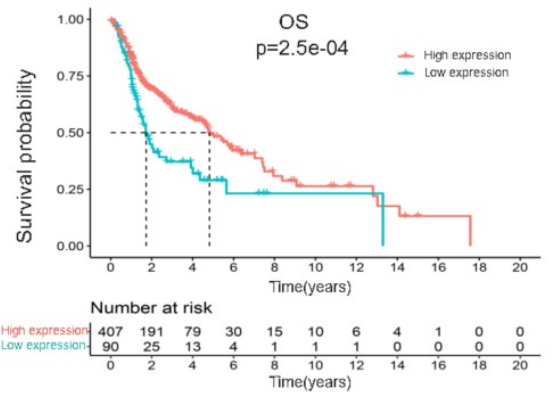

E

miR-3187-3p 3'- GGCGCGUCGGGGUACCGGUU -5' ||| ||||||

PER2-WT 5'- GTCTGCATTCTTATGGCCAT -3'

PER2-Mut 5'- GTCACCTTAGAATACCGGTT -3'
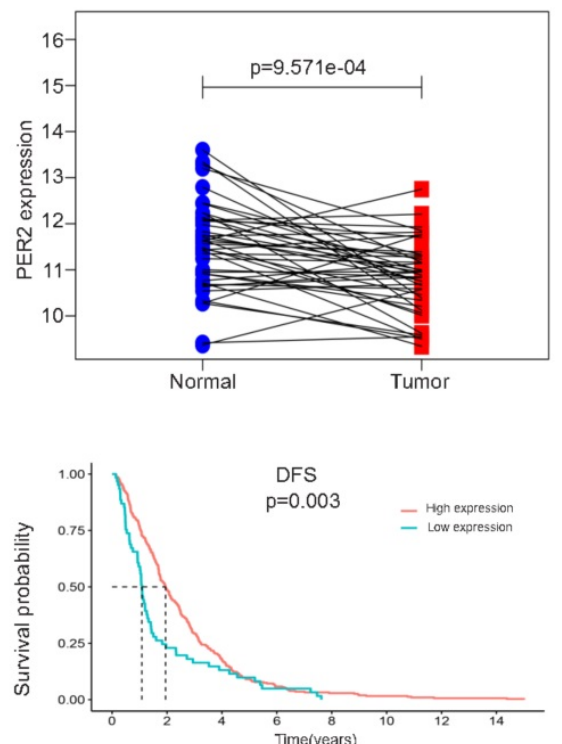

Number at risk

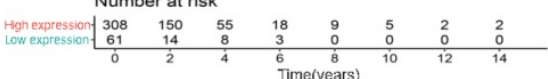

$\mathbf{F}$

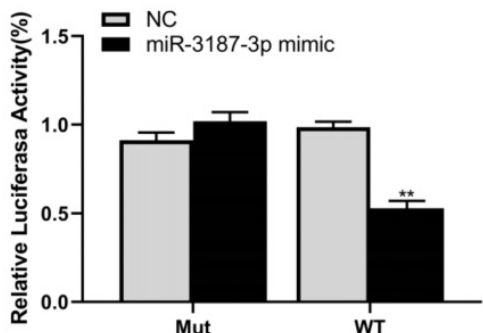

Figure 5. PER2 is a direct target of miR-3187-3p in HNSCCs. A. 12 candidate genes were identified through integrating the results of five algorithms (TargetScan, micro-T-CDS, miRDB, miRTarBase and miRWalk). B. Relative mRNA expression of candidate genes in Fadu, Tca8113 and JHU011 cell lines were detected using qRT-PCR after transfected with miR-3187-3p mimic or inhibitor. C. The expression of PER2 in 502 HNSCCs tissues and 44 adjacent normal tissues, and 43 paired HNSCCs and adjacent non-cancerous samples from the TCGA database were shown. D. Kaplan-Meier curves of OS and DFS in patients with different expression levels of PER2 were shown (Best cutoff $=9.7$ ). E. The binding sequence of miR-3187-3p and PER2 wild-type (WT), and the mutated (Mut) constructs of PER2 was shown. F. Luciferase reporter assays conducted in Fadu cell line demonstrated that PER2 was a direct binding target of miR-3187-3p. The luciferase activity was normalized to the corresponding NC. Data are presented as the mean \pm SD. Student's unpaired t-test, ${ }^{* *}, \mathrm{P}<0.01$.

\section{PER2 mediates the effect of miR-3187-3p on HNSCCs migration and invasion}

To ascertain if PER2 is functionally involved in HNSCCs migration and invasion mediated by miR-3187-3p, initially, the Fadu and Tca8113 cell lines were transfected with PER2 plasmids and an apparent
PER2 overexpression was observed subsequently in both cell lines (Fig. 6A).Then, we performed wound healing and transwell assay to evaluate the changes in invasion and migration ability after upregulated PER2 expression in HNSCCs cell lines and the results indicated that upregulation of PER2 significantly inhibited migration and invasion in Fadu and Tca8113 
cells (Fig. 6B, C). Apart from that, PER2 promotion also obviously decreased the protein levels of $\beta$-catenin, p-GSK-3 $\beta$ and c-Myc in cell lines described above (Fig. 6D). Finally, rescue experiments were conducted to confirm the role of PER2 on the miR-3187-3p-modulated migration and invasion in HNSCCs. PER2 plasmids were next transfected to Fadu and Tca8113 cells to antagonize the miR-3187-3p-mimic-induced hypoactive expression of PER2. As a result, the promotion effect of miR-3187-3p on HNSCCs migration and invasion were relieved by PER2 (Fig. 6E, F). Totally, our data clearly demonstrated that PER2 was involved in miR-3187-3p-mediated migration and invasion.

\section{Discussion}

In the study, we identified that miR-3187-3p, overexpressed in tumor tissue, was related to the aggressive clinical features and poor prognosis of HNSCCs patients in data from TCGA, GEO and our tissue samples cohort. Further a series of in vitro experiments confirmed that miR-3187-3p promoted the migration and invasion of HNSCCs through targeting PER2, accompanied by the activation of Wnt / $\beta$-catenin signaling pathway, which deepened our understanding of the complicated mechanisms of

A

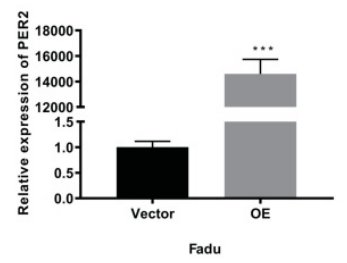

C
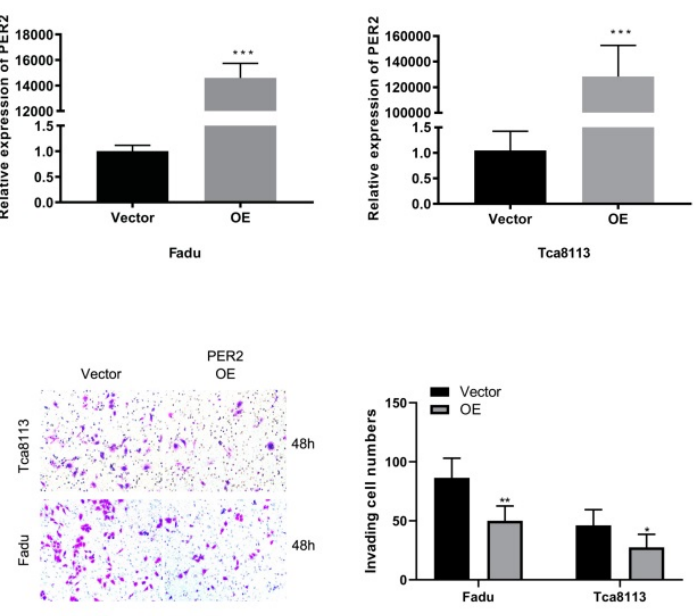

B
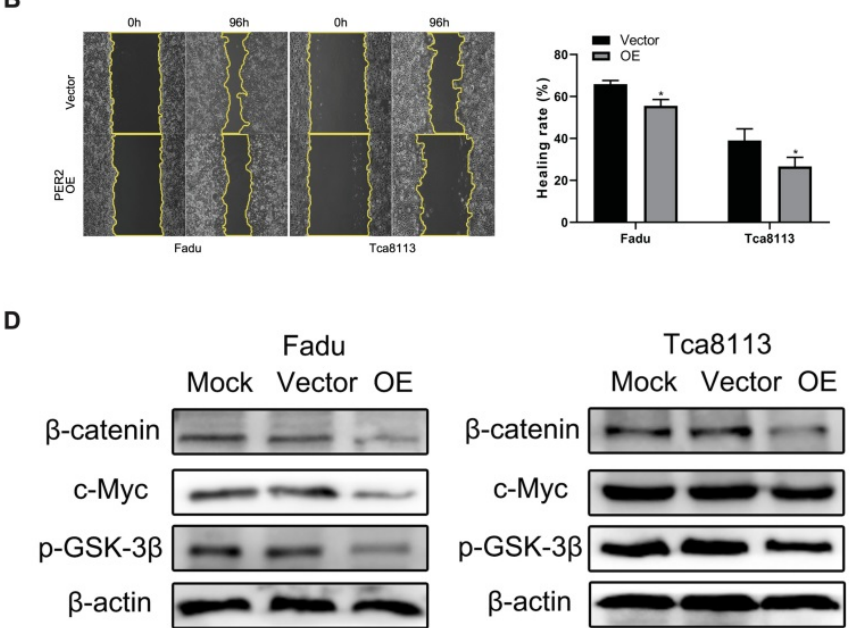

D
E

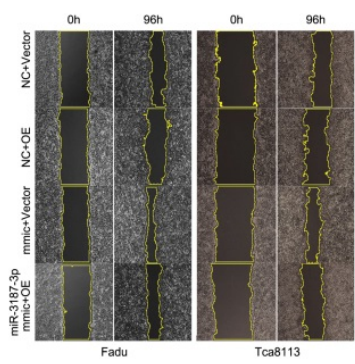

migration and invasion in HNSCCs.

The latest data showed that HNSCCs was the seventh most common cancer in the world, causing approximately 450,000 deaths per year and tumor invasion and metastasis accounted for the primary causes of poor survival in HNSCCs patients [24]. Besides, a pile of literatures had verified that miRNAs were concerned with the invasion and metastasis of diverse cancers [25-28], including HNSCCs [29-32].

Previously, we screened and identified an important $\mathrm{N}$ stage-related miRNA, miR-3187-3p, in HNSCCs by bioinformatic analysis [16], and miR-3187-3p has been confirmed to be a cancer-promoting in several other cancers. For example, miR-3187-3p could promote colorectal cancer proliferation through targeting ASXL1 [33], and upregulated miR-3187-3p expression was associated with enhanced tumor migration and invasion in non-small cell lung cancer [18], and salivary miR-3187-3p could facilitate tumor immune escape in melanoma [34]. Our current study suggested that miR-3187-3p could enhance HNSCCs migration and invasion in vitro with the activation of Wnt / $\beta$-catenin pathway, which might be a novel potential therapeutic targets for improvement of HNSCCs patient outcomes.

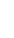
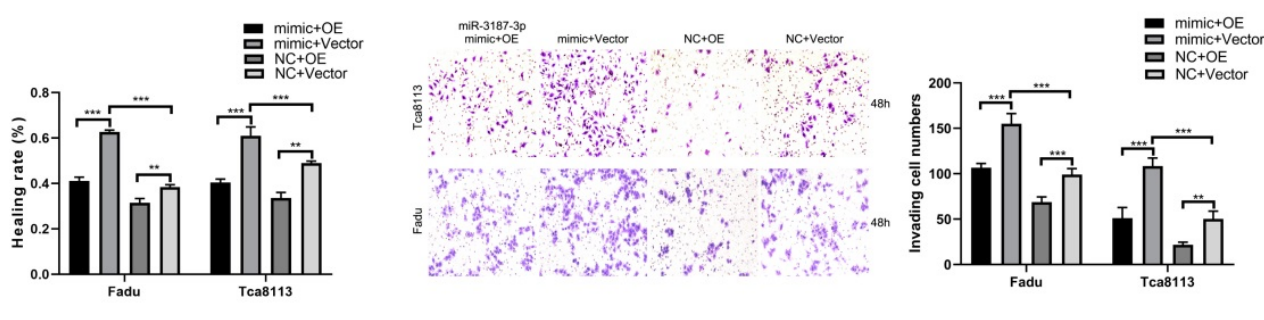

Figure 6. PER2 mediates the effect of miR-3187-3p on HNSCCs migration and invasion. A. PER2 mRNA expression significantly upregulated in Fadu and Tca8113 cell lines after transfection of PER2 plasmids. B, C. Following PER2 overexpression in Fadu and Tca8113 cells, wound-healing (B) and transwell (C) experiment were carried out. D. Western blotting showed that the expression of $\beta$-catenin, P-GSK-3 $\beta$ and c-Myc obviously downregulated in Fadu and Tca8113 cells transfected with PER2 plasmids. E, F. Fadu and Tca8113 Cells transfected with miR-3187-3p mimic or NC were subsequently disposed with PER2 or empty plasmid. Wound healing (E) and transwell staining (F) were evaluated in both cell lines. OE, PER2 overexpression. Data are presented as the mean \pm SD. Student's unpaired t-test, $*, P<0.05 ; * *, P<0.01 ; * * *, P<0.001$. 
Wnt / $\beta$-catenin signaling pathway was one of the key pathway in regulating cell proliferation and differentiation, playing a critical role in tumor invasion and metastasis [19, 21, 22, 35]. $\beta$-Catenin was the core signal transduction in this signaling pathway, activation of which would give rise to high expression of downstream target genes [36]. GSK-3 $\beta$ was a main inhibitory protein of the Wnt / $\beta$-catenin pathway, causing the degradation by ubiquitination of $\beta$-catenin. The phosphorylation of GSK-3 $\beta$ (p-GSK-3 $\beta$ ) would lead to its inactivation, which contributed to the accumulation of $\beta$-Catenin and subsequently activation of downstream target genes, including c-Myc [23]. C-Myc was a proto-oncogene and had been reported to participating in the regulation of tumor progression and invasion in oral squamous cell carcinoma [37]. Therefore, in our present study, miR-3187-3p may enhance the invasive potential of HNSCCs via the activation of Wnt / $\beta$-catenin signaling pathway with the upregulation of $\beta$-catenin, p-GSK-3 $\beta$, and c-Myc.

MiRNAs exerted their functions by silencing the target genes, and it was well-established that a miRNA can bind and regulate multiple target genes, affecting various malignant behaviors of cancer cell [38]. In the study, we identified PER2 as the direct target gene of miR-3187-3p and further analysis of TCGA data indicated that miR-3187-3p and PER2 exhibited a reversed expression state in HNSCCs samples, and high expression of PER2 correlated to a better prognosis in HNSCCs patients as well. PER2 was a well-known clock gene, regulating circadian rhythm in human beings, whose downregulation was related with tumor initiation and progression in various cancers, including HNSCCs [39-42]. It has been reported that PER2 inhibited cell invasion and migration in human oral squamous cell carcinoma [43]. In this study, we found that PER2 overexpression inhibited HNSCCs cells migration and invasion in vitro, showing that PER2 served as a negative regulator in HNSCCs metastasis, consistent with the previous reports. We also identified that upregulation of PER2 attenuated migration and invasion ability of HNSCCs induced by miR-3187-3p, implying that PER2 may be a main target of miR-3187-3p. Additionally, PER2 overexpression also proved to be positive correlated to the expression of Wnt / $\beta$-catenin pathway, which was compatible with previous report in glioblastoma [44].

In conclusion, our study support that high expression of miR-3187-3p is strongly linked to the malignant traits of HNSCCs. High expression of miR-3187-3p predicts a poor outcome in patients with HNSCCs. Moreover, a series of in vitro evidence suggest that miR-3187-3p can directly target tumor suppressor gene PER2, promoting migration and invasion of HNSCCs by activating the Wnt / $\beta$-catenin pathway. Overall, this study illustrates that the miR-3187-3p / PER2 axis may provide a novel promising molecular treatment strategy to subdue the progression of HNSCCs. In future studies, we will investigate how miR-3187-3p / PER2 axis is involved in the HNSCC metastasis in animal models and explore the potential value of this axis as a therapeutic window.

\section{Supplementary Material}

Supplementary figure and table. http://www.jcancer.org/v12p5231s1.pdf

\section{Acknowledgements}

This study was supported by the National Natural Science Foundation of China (Nos. 81773243, 81974424, 81874133, 81772903 and 82073009), the Natural Science Foundation of Hunan Province (Nos. 2019JJ40481 and 2019JJ50944), the Huxiang Young Talent Project (No. 2018RS3024) and the Youth Science Foundation of Xiangya Hospital (No. 2020Q03).

\section{Competing Interests}

The authors have declared that no competing interest exists.

\section{References}

1. Leemans CR, Snijders PJF, Brakenhoff RH. The molecular landscape of head and neck cancer. Nat Rev Cancer. 2018; 18: 269-82.

2. Cramer JD, Burtness B, Le QT, Ferris RL. The changing therapeutic landscape of head and neck cancer. Nat Rev Clin Oncol. 2019; 16: 669-83.

3. Lang L, Shay C, Xiong Y, Thakkar P, Chemmalakuzhy R, Wang X, et al. Combating head and neck cancer metastases by targeting Src using multifunctional nanoparticle-based saracatinib. J Hematol Oncol. 2018; 11: 85.

4. McBride S, Sherman E, Tsai CJ, Baxi S, Aghalar J, Eng J, et al. Randomized Phase II Trial of Nivolumab With Stereotactic Body Radiotherapy Versus Nivolumab Alone in Metastatic Head and Neck Squamous Cell Carcinoma. J Clin Oncol. 2021; 39: 30-7.

5. Burtness B, Harrington KJ, Greil R, Soulieres D, Tahara M, de Castro G, Jr., et al. Pembrolizumab alone or with chemotherapy versus cetuximab with chemotherapy for recurrent or metastatic squamous cell carcinoma of the head and neck (KEYNOTE-048): a randomised, open-label, phase 3 study. Lancet. 2019; 394: 1915-28.

6. Lee RC, Feinbaum RL, Ambros V. The C. elegans heterochronic gene lin-4 encodes small RNAs with antisense complementarity to lin-14. Cell. 1993; 75: 843-54.

7. Wessels HH, Lebedeva S, Hirsekorn A, Wurmus R, Akalin A, Mukherjee N, et al. Global identification of functional microRNA-mRNA interactions in Drosophila. Nature communications. 2019; 10: 1626.

8. Ha M, Kim VN Regulation of microRNA biogenesis. Nature reviews Molecular cell biology. 2014; 15: 509-24.

9. Wang B, Zheng J, Li R, Tian Y, Lin J, Liang Y, et al. Long noncoding RNA LINC02582 acts downstream of miR-200c to promote radioresistance through CHK1 in breast cancer cells. Cell Death Dis. 2019; 10: 764

10. Yu Y, Luo W, Yang ZJ, Chi JR, Li YR, Ding Y, et al. miR-190 suppresses breast cancer metastasis by regulation of TGF-beta-induced epithelial-mesenchymal transition. Mol Cancer. 2018; 17: 70.

11. Huang Q, Xia J, Wang L, Wang X, Ma X, Deng Q, et al. miR-153 suppresses IDO1 expression and enhances CAR T cell immunotherapy. J Hematol Oncol. 2018; 11: 58.

12. Liu Y, Liu R, Yang F, Cheng R, Chen X, Cui S, et al. miR-19a promotes colorectal cancer proliferation and migration by targeting TIA1. Mol Cancer. 2017; 16: 53.

13. Li X, Zhong M, Wang J, Wang L, Lin Z, Cao Z, et al. miR-301a promotes lung tumorigenesis by suppressing Runx3. Mol Cancer. 2019; 18: 99. 
14. Li G, Liu Y, Su Z, Ren S, Zhu G, Tian Y, et al. MicroRNA-324-3p regulates nasopharyngeal carcinoma radioresistance by directly targeting WNT2B. European journal of cancer (Oxford, England : 1990). 2013; 49: 2596-607.

15. Zhang S, He Y, Liu C, Li G, Lu S, Jing Q, et al. miR-93-5p enhances migration and invasion by targeting RGMB in squamous cell carcinoma of the head and neck. J Cancer. 2020; 11: 3871-81.

16. Zhang S, Li G, Liu C, Lu S, Jing $Q$, Chen $X$, et al. miR-30e-5p represses angiogenesis and metastasis by directly targeting AEG-1 in squamous cell carcinoma of the head and neck. Cancer science. 2020; 111: 356-68.

17. Yang G, Zhang T, Ye J, Yang J, Chen C, Cai S, et al. Circ-ITGA7 sponges miR-3187-3p to upregulate ASXL1, suppressing colorectal cancer proliferation. Cancer management and research. 2019; 11: 6499-509.

18. Zhang D, Cai X, Cai S, Chen W, Hu C. Long Intergenic Non-Protein Coding RNA 01089 Weakens Tumor Proliferation, Migration, and Invasion by Sponging miR-3187-3p in Non-Small Cell Lung Cancer. Cancer management and research. 2020; 12: 12151-62.

19. Cai J, Fang L, Huang $Y, \mathrm{Li} R, \mathrm{Xu} X, \mathrm{Hu} Z$, et al. Simultaneous overactivation of Wnt/beta-catenin and TGFbeta signalling by miR-128-3p confers chemoresistance-associated metastasis in NSCLC. Nature communications. 2017; 8: 15870

20. Levrero M, Zucman-Rossi J. Mechanisms of HBV-induced hepatocellular carcinoma. Journal of hepatology. 2016; 64: S84-S101.

21. Sinnberg T, Levesque MP, Krochmann J, Cheng PF, Ikenberg K, Meraz-Torres $\mathrm{F}$, et al. Wnt-signaling enhances neural crest migration of melanoma cells and induces an invasive phenotype. Mol Cancer. 2018; 17: 59.

22. Yang S, Liu Y, Li MY, Ng CSH, Yang SL, Wang S, et al. FOXP3 promotes tumor growth and metastasis by activating Wnt/beta-catenin signaling pathway and EMT in non-small cell lung cancer. Mol Cancer. 2017; 16: 124.

23. Zhan T, Rindtorff N, Boutros M. Wnt signaling in cancer. Oncogene. 2017; 36: 1461-73.

24. Chow LQM. Head and Neck Cancer. The New England journal of medicine. 2020; 382: 60-72.

25. Cao MQ, You AB, Zhu XD, Zhang W, Zhang YY, Zhang SZ, et al. miR-182-5p promotes hepatocellular carcinoma progression by repressing FOXO3a. J Hematol Oncol. 2018; 11: 12.

26. Li Y, Zhang H, Fan L, Mou J, Yin Y, Peng C, et al. MiR-629-5p promotes the invasion of lung adenocarcinoma via increasing both tumor cell invasion and endothelial cell permeability. Oncogene. 2020; 39: 3473-88.

27. Lu Y, Qin T, Li J, Wang L, Zhang Q, Jiang Z, et al. MicroRNA-140-5p inhibits invasion and angiogenesis through targeting VEGF-A in breast cancer. Cancer gene therapy. 2017; 24: 386-92.

28. Yan L, Cai K, Sun K, Gui J, Liang J. MiR-1290 promotes proliferation, migration, and invasion of glioma cells by targeting LHX6. Journal of cellular physiology. 2018; 233: 6621-9.

29. Zhu HM, Jiang XS, Li HZ, Oian LX, Du MY, Lu ZW, et al. miR-184 Inhibits Tumor Invasion, Migration and Metastasis in Nasopharyngeal Carcinoma by Targeting Notch2. Cellular physiology and biochemistry : international journal of experimental cellular physiology, biochemistry, and pharmacology. 2018; 49: 1564-76.

30. Jimenez L, Jayakar SK, Ow TJ, Segall JE. Mechanisms of Invasion in Head and Neck Cancer. Archives of pathology \& laboratory medicine. 2015; 139: 1334-48.

31. Saleh AD, Cheng H, Martin SE, Si H, Ormanoglu P, Carlson S, et al. Integrated Genomic and Functional microRNA Analysis Identifies miR-30-5p as a Tumor Suppressor and Potential Therapeutic Nanomedicine in Head and Neck Cancer. Clinical cancer research : an official journal of the American Association for Cancer Research. 2019; 25: 2860-73.

32. Liu C, Li G, Yang N, Su Z, Zhang S, Deng T, et al. miR-324-3p suppresses migration and invasion by targeting WNT2B in nasopharyngeal carcinoma. Cancer cell international. 2017; 17: 2.

33. Yang G, Zhang T, Ye J, Yang J, Chen C, Cai S, et al. Circ-ITGA7 sponges miR-3187-3p to upregulate ASXL1, suppressing colorectal cancer proliferation. Cancer management and research. 2019; 11: 6499-509.

34. Vignard V, Labbe M, Marec N, Andre-Gregoire G, Jouand N, Fonteneau JF, et al. MicroRNAs in Tumor Exosomes Drive Immune Escape in Melanoma. Cancer immunology research. 2020; 8: 255-67.

35. Zingg D, Debbache J, Pena-Hernandez R, Antunes AT, Schaefer SM, Cheng $\mathrm{PF}$, et al. EZH2-Mediated Primary Cilium Deconstruction Drives Metastatic Melanoma Formation. Cancer cell. 2018; 34: 69-84 e14.

36. Jing $\mathrm{Q}, \mathrm{Li} \mathrm{G}$, Chen $\mathrm{X}$, Liu C, Lu S, Zheng $\mathrm{H}$, et al. Wnt3a promotes radioresistance via autophagy in squamous cell carcinoma of the head and neck. Journal of cellular and molecular medicine. 2019; 23: 4711-22.

37. Li S, Zhang S, Chen J. c-Myc induced upregulation of long non-coding RNA SNHG16 enhances progression and carcinogenesis in oral squamous cell carcinoma. Cancer gene therapy. 2019; 26: 400-10.

38. Hammond SM. An overview of microRNAs. Advanced drug delivery reviews. 2015; 87: 3-14.

39. Miki T, Matsumoto T, Zhao Z, Lee CC. p53 regulates Period2 expression and the circadian clock. Nature communications. 2013; 4: 2444.

40. Hwang-Verslues WW, Chang PH, Jeng YM, Kuo WH, Chiang PH, Chang YC, et al. Loss of corepressor PER2 under hypoxia up-regulates OCT1-mediated EMT gene expression and enhances tumor malignancy. Proceedings of the National Academy of Sciences of the United States of America. 2013; 110: 12331-6.
41. Papagiannakopoulos T, Bauer MR, Davidson SM, Heimann M, Subbaraj L, Bhutkar A, et al. Circadian Rhythm Disruption Promotes Lung Tumorigenesis. Cell metabolism. 2016; 24: 324-31.

42. Xiang R, Cui Y, Wang Y, Xie T, Yang X, Wang Z, et al. Circadian clock gene Per2 downregulation in nonsmall cell lung cancer is associated with tumour progression and metastasis. Oncology reports. 2018; 40: 3040-8.

43. Su X, Chen D, Yang K, Zhao Q, Zhao D, Lv X, et al. The circadian clock gene PER2 plays an important role in tumor suppression through regulating tumor-associated genes in human oral squamous cell carcinoma. Oncology reports. 2017; 38: 472-80.

44. Ma D, Hou L, Xia H, Li H, Fan H, Jia X, et al. PER2 inhibits proliferation and stemness of glioma stem cells via the Wnt/betacatenin signaling pathway. Oncology reports. 2020; 44: 533-42. 